Article

\title{
Preparation, Characterization and Performance Properties of Polydodecylmethylsilsesquioxane Nanoparticles
}

\author{
Fuquan Deng ${ }^{1,2}$, Hua Jin ${ }^{3}$ and Wei $\mathrm{Xu}^{2,4, *}$ \\ 1 College of Art and Design, Shanxi University of Science and Technology, Xi'an 710021, China; \\ dengfuquan@sust.edu.cn \\ 2 Zhejiang Wenzhou Research Institute of Light Industry, Wenzhou 325003, China \\ 3 Wenzhou Vocational and Technical College, Wenzhou 325035, China; jh-skd@163.com \\ 4 College of Bioresources Chemical and Materials Engineering, Shaanxi University of Science and Technology, \\ Xi'an 710021, China \\ * Correspondence: xwforward@163.com or xuwei@sust.edu.cn; Tel.: +86-29-8616-8235
}

Received: 8 October 2020; Accepted: 27 October 2020; Published: 29 October 2020

\begin{abstract}
A series of polydodecylmethylsilsesquioxane (PDMSQ) nanocomposite latexes were prepared via emulsion polymerization of methyltriethoxysilane (MTES) and dodecyltrimethoxysilane (DTMS) and sodium hydroxide as the catalyst, and sodium dodecyl benzene sulfonate/Tween 80 as the mixed emulsifiers. Effects of the emulsifier doses, the reaction temperature, the catalyst concentration and the oil/water ratio on the particle size and distribution of the PDMSQ nanoparticles were discussed. Particle size and micromorphology, structure, thermal stability, crystallinity and hydrophobicity of PDMSQ nanoparticles (PDMSQ NPs) were investigated by dynamic laser scattering (DLS), Fourier transform infrared spectroscopy (FTIR), silicon-nuclear magnetic resonance $\left({ }^{28} \mathrm{Si}-\mathrm{NMR}\right), \mathrm{X}$-ray photoelectron spectroscope (XPS), scanning electron microscope (SEM), transmission electron microscope (TEM), atomic force microscope (AFM), thermo gravimetric analysis (TGA), X-ray diffraction (XRD) and contact angle tester. Results showed that a series of PDMSQ NPs could be obtained with an average particle size of less than $80 \mathrm{~nm}$ and narrow distribution as well as spherical structure under the optimal process conditions. PDMSQ NPs exhibited excellent thermal stability and were mainly amorphous but also contained some crystal structures. Importantly, the static water contact angles (WCAs) on its latex films were larger than $150^{\circ}$ and the WCAs hysteresis were less than $10^{\circ}$, thus those PDMSQ nanocomposite latexes show potential in the field of superhydrophobic coatings.
\end{abstract}

Keywords: polysilsesquioxane; nano-hybrid; film morphology; hydrophobicity

\section{Introduction}

Functional polysilsesquioxane nanoparticles (PSQ NPs) are a new kind of important hybrid material [1]. They are provided with the unique surface effect, heat stability, flame retardance, oxidation resistance, physiological inertia and high strength that the traditional inorganic nanomaterials, such as $\mathrm{SiO}_{2}, \mathrm{TiO}_{2}, \mathrm{ZnO}$, etc., usually do not possess. Moreover, the organic groups in their molecules are easy to be modified and structurally functionalized, which can achieve real molecule-level composites with the polymer matrixes [2,3]. Therefore, in recent years functional polysilsesquioxane nano-hybrid materials have found broad application prospects in photoluminescent materials, photonic crystals, adsorption materials, biomedical carriers, flame retardant materials, functional coatings and electrochromic materials $[2,4,5]$. 
Hitherto, PSQ NPs are mainly synthesized by the hydrolysis-condensation reaction of trialkoxy silanes bearing different organic moieties $\left[\mathrm{R}-\mathrm{Si}\left(\mathrm{OCH}_{3}\right)_{3}\right.$ or $\left.\mathrm{R}-\mathrm{Si}\left(\mathrm{OC}_{2} \mathrm{H}_{5}\right)_{3}\right]$. Normally, those reactions can be held in the three diverse media, i.e., emulsion, water and alcohol/water mixed systems, among which the emulsion system is often chosen to prepare the PSQ NPs as a result of some advantages such as mild reaction conditions and lower reaction temperature, controllable size and narrow size distribution of PSQ NPs as well as high regularity of spheres [6]. According to the sort of the different organic groups in PSQ NPs, those can be divided into monofunctional polysilsesquioxanes and multifunctional polysilsesquioxanes, mainly bifunctional polysilsesquioxanes. By contrast, bifunctional polysilsesquioxanes have some more advantages in structural design and functionality regulation. Therefore, much attention has been paid to those bifunctional PSQ in recent years, e.g., Kim's groups utilized methyltrimethoxysilane as a precursor and a simple one-step hydrolytic co-condensation method to prepare a series of functional polysilsesquioxanes nano/micro hard spheres such as polymercaptopropylmethylsilsesquioxane, polymethacryloxypropylmethylsilsesquioxane, polyvinylmethylsilsesquioxane, polyaminopropylmethylsilsesquioxane, and polyphenylmethylsilsesquioxane [7]. In our previous work [8], a series of monodispersed polyaminopropylmethylsilsesquioxane nanospheres were also prepared through emulsion polymerization of methyltriethoxysilane in the mixed solutions of sodium hydroxide, sodium dodecylsulfonate/fatty alcohol-polyoxyethylene ether and $\gamma$-aminopropyltriethoxysilane as a modifier. Ottenbrite et al. used a self-catalyzed method, employing aminopropyltriethoxysilane as the catalyst to synthesize polyaminopropylvinylsilsesquioxane nanoparticles [9]. Kim et al. prepared several polysilsesquioxane particles bearing bulky UV chromophores, under basic conditions, that are potentially useful as UV blocking ingredients in sunscreens [4]. Mori et al. prepared a sort of amphiphilic polysilsesquioxanes hybrids through the hydrolytic co-condensation reaction in homogeneous $\mathrm{N}, \mathrm{N}$-dimethylformamide (DMF) solutions, where fluorinated triethoxysilane and hydroxyl-functionalized triethoxysilane were used as raw materials [10]. In addition, a kind of fluorescent and octylene-bridged polysilsesquioxanes nanoparticles were prepared by acid-catalyzed and sol-gel polymerization of 1,8-bis(triethoxysilyl)octane (BESO) [11]. Nevertheless, Lee et al. utilized one step reaction from admixtures of several precursors to prepare the multifunctional polysilsesquioxanes which found a potential application in exfoliater, fillers and carriers of different useful ingredients [12]. In view of the above presentations, polysilsesquioxane nanoparticles can be prepared via precise control of the reaction media, the catalyst and the functional monomer species, and the reaction conditions, to obtain the desired morphology and particle size distribution as well as the requisite functions.

The application of hydrophobic PSQ NPs in functional coatings, such as hydrophobic, antibacterial and self-cleaning coatings, has become a hot topic in the field of nanomaterials. However, there are relatively few reports on this aspect at present. Zou et al. [13] synthesized novel PSQ aerogels via a simple sol-gel reaction where the thiourethane bonds were artfully designed as the bridging groups to tailor the morphology and mechanical performance of these aerogels; finally, the PSQ aerogels films exhibited inherent superhydrophobicity and other kinds of mechanical performance. Ma et al. [14] utilized hydrophobic (methyl or vinyl based) polysilsesquioxane microspheres to successfully construct the durable superhydrophobic coating on cotton fabric surface. In addition, fumed or silane (siloxane)-modified silica nanocomposites could be encountered in some references $[15,16]$ to fabricate the superhydrophobic coatings in combination with PSQ. In addition, in Dong's and Yuan's works [1,17], polysilsesquioxane/polystyrene microspheres and polysilsesquioxane/polyamine nanocomposites were individually fabricated and then those inorganic-organic hybrids could be used to construct the superhydrophobic coatings conveniently. To date, structures of the hydrophobic PSQ NPs are not rich enough and there is still a lack of novel designed PSQ NPs with multi-functions. Furthermore, bi-functional PSQ NPs have more advantages in structure regulation and chemical modification $[7,9]$ and therefore gained more and more attention. 
In consideration of the above, a series of the polydedocylmethylsilsesquioxane nanohybrid emulsions were prepared by emulsion polymerization with MTES and DTEM as silicon sources, sodium dodecyl benzene sulfonate/Tween 80 as mixed emulsifiers and sodium hydroxide as catalyst. Some synthetic process parameters, e.g., the emulsifier dosage, the catalyst dosage, the reaction temperature and the oil/water ratio, were scrutinized to investigate their effects on the particles' size and distribution, and morphology. In addition, chemical structure, thermal properties, crystallinity and hydrophobicity of the as-prepared PSQ NPs were discussed.

\section{Materials and Methods}

\subsection{Materials}

Methyltriethoxysilane (MTES) and dodecyltrimethoxysilane (DTMS) were analytical reagents purchased from Hubei Wuda Organic Silicon New Chemical Co., Ltd., Wuhan, China. Sodium dodecyl benzene sulfonate (SDBS), analytical pure grade, was obtained from Zhengzhou Tusheng Chemical Co., Ltd., Zhengzhou, China. Tween 80, chemically pure grade, was bought from Tianjin Tianli Chemical Reagent Co., Ltd., Tianjin, China. Sodium hydroxide was of chemical grade and acquired from Sinopharm Group Chemical Reagent Co. Ltd., Shanghai, China. The above reagents were directly used without any pretreatment. The Si wafers were purchased from Shanghai Songjiang Silicon Material Co., Ltd., Shanghai, China and were used in fine morphology detection. Before use, those samples were rinsed and disposed on the basis of our previous works $[18,19]$.

\subsection{Preparation of Polydodecylmethylsilsesquioxane (PDMSQ) Nanoparticles Latexes}

In a $250 \mathrm{~mL}$ three-necked flask equipped with an agitator, thermometer and reflux condensing tube, $100 \mathrm{~g} \mathrm{H}_{2} \mathrm{O}$ and an appropriate amount of the mixed emulsifiers (SDBS/Tween 80) and the sodium hydroxide liquors were dropped successively according to the stoichiometric recipes. Then, those above mixtures were fully stirred to the homogeneous state under the different established temperatures. Next, $6.80 \mathrm{~g}$ of MTES was slowly dropped into the mixed system which then turned into a bluish translucent emulsion. A quantity of $1.50 \mathrm{~g}$ of DTMS was dropwise added into the compound after the MTES solution was finished. While all of those were accomplished, the system was kept stirring for another $12 \mathrm{~h}$. Finally, the translucent emulsion was obtained, i.e., the polydodecylmethylsilsesquioxane nanoparticles latex was successfully prepared. The detailed recipes and relevant parameters of the PDMSQ NPs latexes preparation are listed in Table 1.

Table 1. Recipes and relevant parameters of the PDMSQ NPs latexes preparation.

\begin{tabular}{|c|c|c|c|c|c|c|c|}
\hline $\begin{array}{l}\text { Sample } \\
\text { Number }\end{array}$ & $\begin{array}{l}\text { Mass of } \\
\text { MTES (g) }\end{array}$ & $\begin{array}{c}\text { Mass of } \\
\text { DTMS (g) }\end{array}$ & $\begin{array}{c}\text { Mass of } \\
\mathrm{NaOH}(\mathrm{g})\end{array}$ & $\begin{array}{l}\text { Mass of } \\
\text { SDBS (g) }\end{array}$ & $\begin{array}{c}\text { Mass of } \\
\text { Tween } 80(\mathrm{~g})\end{array}$ & $\begin{array}{l}\text { Mass of } \\
\mathrm{H}_{2} \mathrm{O}(\mathrm{g})\end{array}$ & $\begin{array}{c}\text { Reaction } \\
\text { Temperature }\left({ }^{\circ} \mathrm{C}\right)\end{array}$ \\
\hline P1 & 6.80 & 1.50 & 0.20 & 0.18 & 0.18 & 100 & 60 \\
\hline P2 & 6.80 & 1.50 & 0.20 & 0.27 & 0.27 & 100 & 60 \\
\hline P3(P8) & 6.80 & 1.50 & 0.20 & 0.36 & 0.36 & 100 & 60 \\
\hline $\mathrm{P} 4$ & 6.80 & 1.50 & 0.20 & 0.45 & 0.45 & 100 & 60 \\
\hline P5 & 6.80 & 1.50 & 0.20 & 0.54 & 0.54 & 100 & 60 \\
\hline P6 & 6.80 & 1.50 & 0.20 & 0.36 & 0.36 & 100 & 40 \\
\hline P7 & 6.80 & 1.50 & 0.20 & 0.36 & 0.36 & 100 & 50 \\
\hline P8(P3) & 6.80 & 1.50 & 0.20 & 0.36 & 0.36 & 100 & 60 \\
\hline P9 & 6.80 & 1.50 & 0.20 & 0.36 & 0.36 & 100 & 65 \\
\hline P10 & 6.80 & 1.50 & 0.20 & 0.36 & 0.36 & 100 & 70 \\
\hline
\end{tabular}

Note: Px denotes the sample number of the PDMSQ NPs latexes.

\subsection{Preparation of the PDMSQ Nanoparticles}

An appropriate amount of the PDMSQ latex was successively filtrated, washed and dried under the reduced pressure to obtain the solid sample. Then, it was ground into the fine PDMSQ powder. 


\subsection{Characterization}

The PDMSQ latexes were diluted to the solid content of $0.2 \mathrm{wt} . \%-0.5 \mathrm{wt} . \%$ and then dropped onto a slice of monosilicon wafer until being dried at ambient temperature. Next, they were kept for $24 \mathrm{~h}$ in a vacuum drying apparatus for balance. Ahead of the observation, the pretreated samples were sputtered with gold under a vacuum and then observed with a scanning electron microscope (SEM, S4800, Hitachi Co., Ltd., Tokyo, Japan).

The PDMSQ latexes were diluted to some suitable extent and then stained with $2 \mathrm{wt} . \%$ phosphotungstic acid liquor. Next, the PDMSQ latex particles were observed and photographed by the transmission electron microscope (TEM, Tecnai G2 F20 S, FEI. Co., Ltd., Hillsboro, OR, USA). Meanwhile, their particles' size and size distribution were also determined by the laser particle size analyzer (Nano-ZS, Malvern Co., Ltd., Whitnash, UK).

The PDMSQ latexes were diluted to the solid content of $0.03 \mathrm{wt} . \%$. Then, a little slice of monosilicon wafer was vertically immersed into the above finishing solution and kept for several seconds to ensure that the PDMSQ molecules were adsorbed onto the silicon surface. Next, it was fetched out and dried for $5 \mathrm{~min}$ at $100^{\circ} \mathrm{C}$, baked for $1 \mathrm{~min}$ at $160^{\circ} \mathrm{C}$ to finalize the sample. Finally, fine morphologies of the PDMSQ particles were detected by atomic force microscope (AFM, Nanoscope IIIA, Digital Instruments Co., Ltd., Tonawanda, NY, USA) in tapping mode where the determining temperature and the ambient relative humidity were directed as $20 \pm 0.5^{\circ} \mathrm{C}$ and $49.5 \% \pm 2 \%$, respectively.

The infrared spectra of the silane monomers, MTES and DTMS, and the final product, PDMSQ, were determined by the $\mathrm{KBr}$ coating method and the $\mathrm{KBr}$ power tablet method, respectively, with a spectrometer (VECTOR-22, Bruker Co., Ltd., Bremen, Germany) in the range of $4000-400 \mathrm{~cm}^{-1}$. The ${ }^{29} \mathrm{Si}-\mathrm{NMR}$ measurement was carried out with a spectrometer (Avance III 400, Bruker Co., Ltd., Bremen, Germany) and glycine specimen was utilized as the external standard.

The determination on the sample elements was implemented by an X-ray photoelectron spectrometer (Axis Supra, Kratos Analytical Co., Ltd., Stretford, UK) in which the monochromatic Al $K \alpha$ rays were utilized as the $X$-ray source, and the angle of incidence and the vacuum value in the analysis room were $90^{\circ}$ and $1.2 \times 10^{-8} \mathrm{~Pa}$, respectively. The spectra line deviation was rectified using the binding energy value $(284.8 \mathrm{eV})$ of the contaminated carbon $\mathrm{C} 1 \mathrm{~s}$ on the sample surface.

Thermal stability of the sample was analyzed by a thermogravimeter (Q500, TA Co., Ltd., New Castle, DE, USA) where the heating rate of $10^{\circ} \mathrm{C} / \mathrm{min}$ was applied under the inert atmosphere and the temperature range was from the ambient temperature to $800^{\circ} \mathrm{C}$.

The PDMSQ nanoparticle was placed in the groove of the sample plate. Then, it was tested with an X-ray diffractometer (D8 Advanced, Bruker Ltd., Bremen, Germany) where $\mathrm{Cu}$ K $\alpha$ radiation $(\alpha=0.154 \mathrm{~nm}$ ) was used with generator settings of $45 \mathrm{~mA}$ and $45 \mathrm{kV}$. The diffraction angle range was $5^{\circ}-60^{\circ}$, the diffraction step size was $0.02^{\circ}$, and the diffraction step was $0.1 \mathrm{~s}$ in continuous mode.

Water contact angles on the PDMSQ nanoparticles loaded glass slides were determined by a contact angle goniometer (OCA20, Dataphysics Ltd., Filderstadt, Germany) where $5 \mu \mathrm{L}$ of the injecting volume was adopted. For diminishing the experimental errors, at least nine repeated determinations were done and their average was used as the final result for one sample.

\section{Results and Discussion}

\subsection{Formation Mechanism of the PDMSQ Nanospheres}

Hydrophobic PDMSQ nanoparticles were prepared by the hydrolysis-condensation reaction of methyl triethoxysilane (MTES) and dodecyl trimethoxysilane (DTMS) in the SDBS/Tween 80 mixed emulsifiers aqueous solution. So, formation mechanism of the PDMSQ nanospheres is mainly composed of the following three procedures, as illustrated in Figure 1: (1) MTES is first hydrolyzed to generate methylsilanol under the action of base catalyst, and then methylsilanol undergoes the self-condensation reaction to form $\mathrm{Si-O}-\mathrm{Si}$ networks, thus many tiny nuclear phase seeds have been formed. (2) DTMS is also hydrolyzed to produce dodecylsilanol which then is co-condensed with seeds 
of nuclear phase. (3) The growth stage of the nuclear phase seeds to the final PDMSQ nanoparticles, i.e., the transfer of monomers from droplets to numerous nuclear phase seeds, persistent condensation reaction and formation of the PDMSQ particles, are finally accomplished.

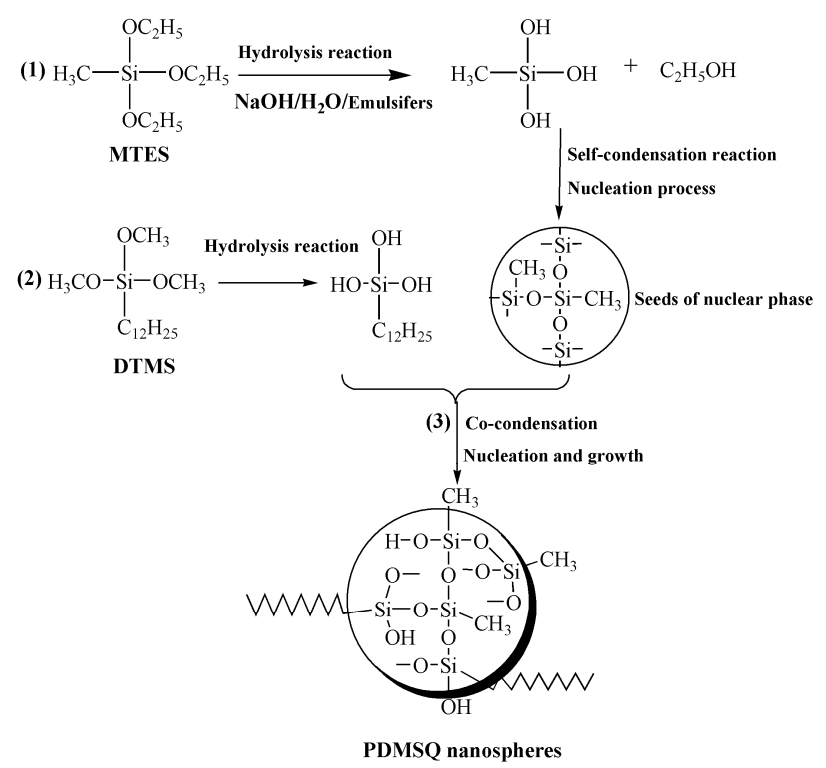

Figure 1. Formation mechanism of PDMSQ NPs.

\subsection{Discussion on Influencing Factors of the Preparation Technology}

Currently, PSQ NPs are mainly synthesized by the hydrolysis-condensation reaction of trialkoxy silanes in different emulsion, water or alcohol/water mixed systems. However, for less water-soluble silanes, the emulsion system is often chosen. In general, the reaction conditions such as the emulsifier dose, the reaction temperature, the catalyst usage and the monomer concentration will influence the average particle size and microstructure of PSQ NPs [7]. Therefore, the mixed emulsifiers' dose, the reaction temperature, the catalyst usage and the monomer/water mass ratio were set as 0.35 $\mathrm{wt} . \%-1.07 \mathrm{wt} . \%, 40-70{ }^{\circ} \mathrm{C}, 25-125 \mathrm{mmol} / \mathrm{L}$ and 1:4-1:20, respectively, and their effect was investigated on the average particle size of PDMSQ NPs by individual factor experiment in this paper. These results are fully presented in Figure 2.

Firstly, the reaction temperature, the concentration of $\mathrm{NaOH}$ and the oil-water mass ratio were immobilized as $60{ }^{\circ} \mathrm{C}, 50 \mathrm{mmol} / \mathrm{L}$ and 1:12, respectively, and the mass concentration of the compound emulsifiers was varied between $0.35 \mathrm{wt} . \%$ and $1.07 \mathrm{wt} . \%$ to explore its effect on the mean particle size and distribution of PDMSQ NPs. The result is shown in Figure 2a. As can be seen, the average particle size and its polydispersity index (PDI) of PDMSQ latex particles rapidly decreased when the compound emulsifier dosage was increased from $0.36 \mathrm{wt} . \%$ to $0.71 \mathrm{wt} . \%$, whereas this downward trend would flatten out and tended to be almost constant when w (SDBS/T80) was greater than $0.71 \mathrm{wt} . \%$. At this time, the mean diameter and PDI of PDMSQ latex particles were all less than $100 \mathrm{~nm}$ and 0.2 , respectively. This experimental consequence is probably related to a mechanism of the micellar nucleation although the hydrolysis-condensation reactions, rather than radical polymerization, are involved in the mixed emulsions for the present experimental system. Specifically, solubilizing micelles containing the silane monomers are the sites of the hydrolysis-condensation reactions, and particle nucleation first starts there. Then, the transfer of monomers from droplets to solubilizing micelles, particles growth and formation of polymer particles are continuously completed as described in reference [20]. Therefore, the less the emulsifier dosage is, the lower the micelles concentration in the system and the fewer number of the nucleation particles are. Moreover, the hydrolysis-condensation reactions will not proceed uniformly under the lower amounts of micelles concentration. In this way, the average particle size and PDI of PDMSQ NPs are relatively large under the same amount of 
monomers. While the emulsifier contents are increased to some extent (larger than $0.71 \mathrm{wt} . \%$ under the current system), the micelles concentration is sufficiently large to prompt the number of nucleation particles to increase rapidly and the polymer particles to grow completely. Thus, the as-prepared PDMSQ NPs possess a relatively small particle size and PDI. Considering these above findings, the optimal mixed emulsifier dosage is chosen as $0.71 \mathrm{wt} . \%$.

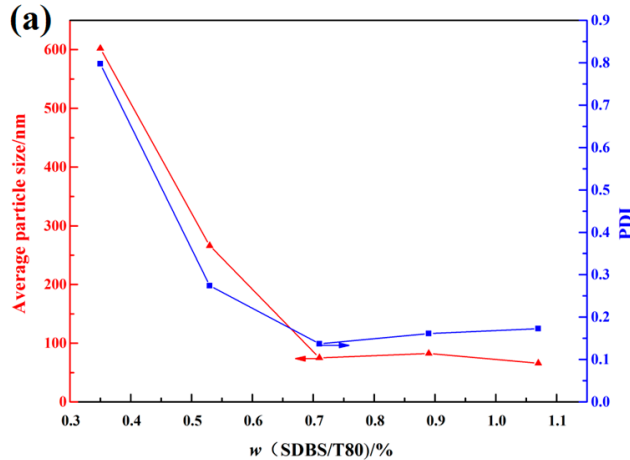

(c)

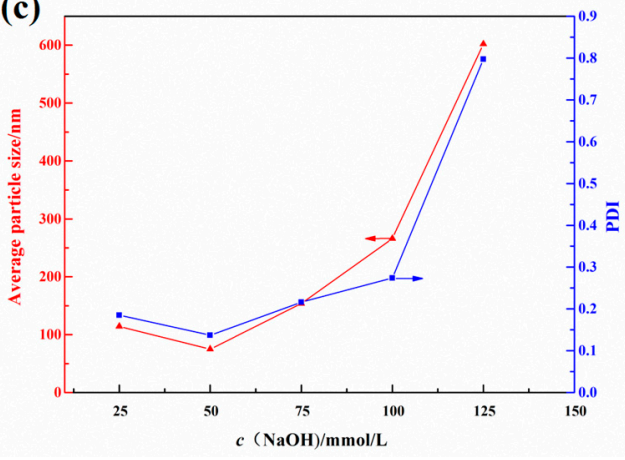

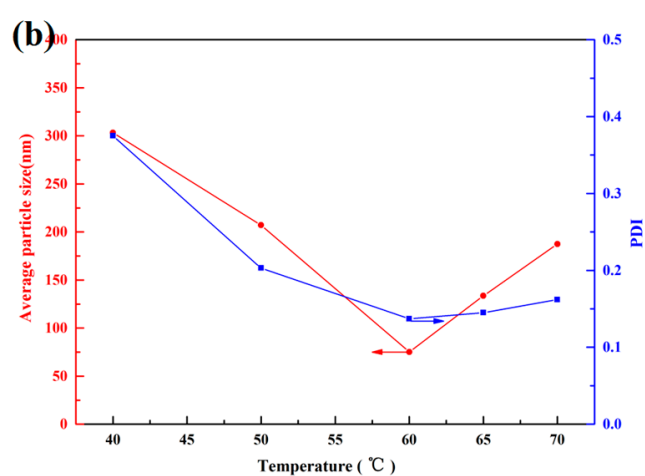

(d)

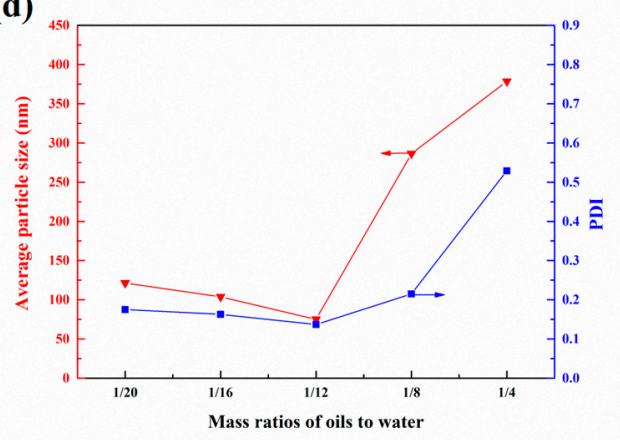

Figure 2. Effect of (a) the emulsifier doses, (b) the reaction temperature, (c) the catalyst concentration and (d) the water/oil mass ratios on the average particle size and its distribution.

Secondly, the mixed emulsifier dosage, the concentration of $\mathrm{NaOH}$ and the oil-water mass ratio were fixed as $0.71 \mathrm{wt} . \%, 50 \mathrm{mmol} / \mathrm{L}$ and 1:12, respectively, and the reaction temperature was changed from 40 to $70{ }^{\circ} \mathrm{C}$ to investigate its effect. The result is shown in Figure $2 \mathrm{~b}$. It can be discovered that the particle size and PDI of the PDMSQ NPs were both first decreased and then increased with the increasing temperature from 40 to $70{ }^{\circ} \mathrm{C}$, and the lowest values of particle size and PDI were obtained while the reaction temperature was $60^{\circ} \mathrm{C}$. Herein, the reaction temperature plays a key role in the hydrolysis-condensation reaction rate of silane monomers and the aggregation state of surfactant molecules, especially the latter which will affect the critical micelle concentration (CMC) of surfactants. That is, it will influence the particle size and distribution of the PDMSQ NPs according to the statement in the previous paragraph. In addition, based on the published literature [21-24], the CMC of pure ionic surfactants or their mixtures with other types of surfactants generally decreases and then increases with the augmentation of the temperature. Khoshnood et al. [25] have also utilized a molecular thermodynamic model to testify the same nonmonotonic behavior of CMC versus temperature. Thus, under the condition of the same emulsifier dosage, the CMC might firstly decrease and then increase with the increasing of temperature. On the contrary, the micelles concentration and the nucleation particles' quantity firstly increased and then decreased. Finally, particle size and distribution of the PDMSQ NPs were first decreased and then increased with the increasing temperature. Furthermore, we know that the Krafft point of sodium dodecyl benzene sulfonate was about $62.5^{\circ} \mathrm{C}$ from Tsujii's work [26]. In consequence, from the perspective of practical application, the optimal reaction temperature is selected as $60^{\circ} \mathrm{C}$. 
Base is a commonly used catalyst for the hydrolysis-condensation of silane, such as ammonia, triethylamine, sodium hydroxide and tetramethylammonium hydroxide. However, the first two are a weak base whereas the latter two are strong bases. It is obvious that MTES and DTMS are neutral and the target products could not be easily obtained by weak base catalyst. Therefore, $\mathrm{NaOH}$ catalyst was chosen in this paper. On basis of the designed program, the result of the $\mathrm{NaOH}$ effect is displayed in Figure 2c. It can be seen that the $\mathrm{NaOH}$ dosage exerted great influence on the average particle size of the PDMSQ NPs. The mean particle size and PDI of the PDMSQ NPs were kept as a small range under the condition of $\mathrm{NaOH}$ concentrations of less than $50 \mathrm{mmol} / \mathrm{L}$, while they would be increased at higher $\mathrm{NaOH}$ concentrations larger than the above value. This is mainly because the amount of sodium hydroxide will directly affect the nucleation and nuclear growth of PDMSQ NPs. At low amounts of base catalyst, both the nucleation process and the nuclear growth process proceeded slowly and thoroughly, therefore, the relatively higher base dosage would help to generate more nuclear phase seeds and finally the mean particle size was less small under the circumstance of the same monomer content. On the contrary, high levels of $\mathrm{NaOH}$ usage would compel a fast speed of the nuclear phase seeds' formation and the nuclear growth, and even produce highly-agglomerated colloidal particles, which lead to the big PDMSQ NPs with large PDI. Thus, the optimal concentration of $\mathrm{NaOH}$ is adopted as $50 \mathrm{mmol} / \mathrm{L}$ in this experiment.

Finally, the effect of the oil-water mass ratio is shown in Figure 2d. It was found that the mean particle size and its PDI of the obtained PDMSQ NPs were relatively small when the oil-water mass ratio was less than 1:12, however, they would be greatly increased when the oil-water mass ratio was more than 1:12. This may be due to the incomplete hydrolysis-condensation reaction of silanols and exposure of highly-active groups on the nanoparticles surface under the high concentration of monomers, which probably result in the agglomerations of those particles. Thus, the preferable oil-water mass ratio was chosen as 1:12.

\subsection{Micromorphology of the PDMSQ Nanoparticles}

SEM and TEM images of the PDMSQ NPs were observed and the results of three representative samples are shown in Figures 3 and 4. It is clearly discovered that the appearance of three samples all emerged as a relatively spherical pattern with uniform size. By estimating from the scale bar, the approximate diameters of the P3, P9 and P10 samples were about 60, 100 and $150 \mathrm{~nm}$, respectively, which are all slightly less than those values determined by the laser particle size analyzer. However, those testing results are basically consistent in view of the latter, which includes the size of the hydrated sheathes on the PDMSQ particles.

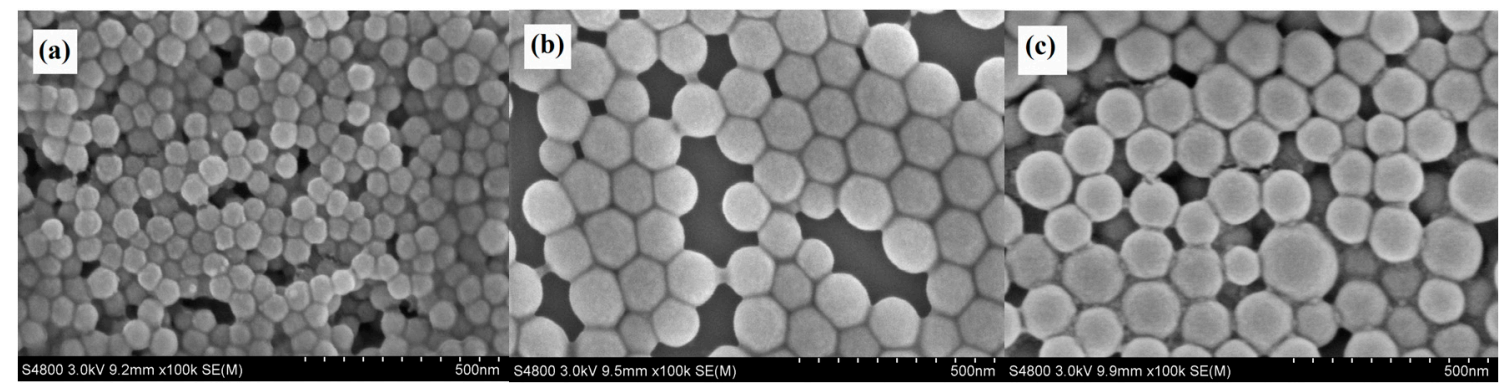

Figure 3. SEM images of the PDMSQ NPs: (a) sample P3, (b) sample P9, and (c) sample P10. 


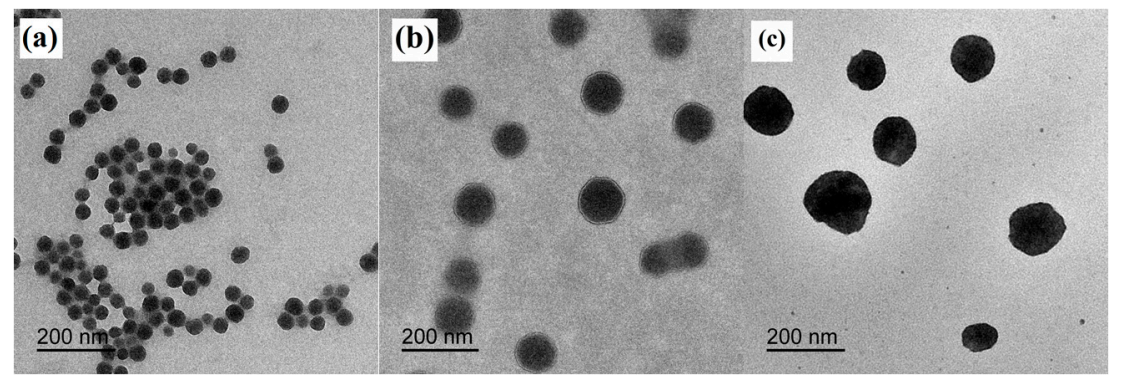

Figure 4. TEM images of the PDMSQ NPs: (a) sample P3, (b) sample P9, and (c) sample P10.

To get a better understanding of the fine morphology of PDMSQ nanospheres, three samples, orderly arranged on the monocrystalline silicon surface, were observed by atomic force probe microscopy. The results are displayed in Figure 5 as AFM topographies. It is clearly discovered on the AFM topographies (within the scanning range of $2 \mu \mathrm{m} \times 2 \mu \mathrm{m}$ and at the observing data scale of $100 \mathrm{~nm}$ ) that there were almost monolayers of closely-arrayed nanospheres on the silicon wafer surface, except sample P9, which showed overlapped layers of the nanospheres, possibly as a result of multilayer adsorption of nanoparticles on silicon wafer. Nanoparticles appeared as regular spheres with monodispersed size and smooth surface. As evaluated from the AFM topographies, mean particle sizes of three PDMSQ samples, P3, P9 and P10, were about 60, 100 and $150 \mathrm{~nm}$ and those results are in accordance with the above SEM, TEM and laser particle size analyzer outcomes.
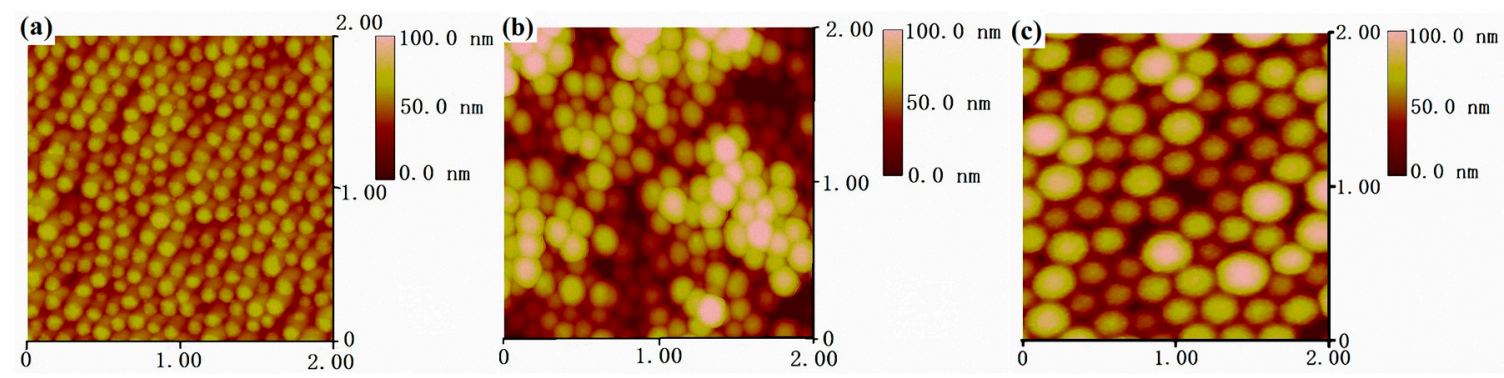

Figure 5. AFM topographies: (a) sample P3, (b) sample P9, and (c) sample P10.

\subsection{Structure Characterization}

FTIR spectra of the reactants and the product were tested and are compared in Figure 6. Obviously, the following absorption peaks were all located in three curves: the characteristic peaks at 2971, 2854, 1476 and $1414 \mathrm{~cm}^{-1}$, and strong absorption peaks at 1275 and $771 \mathrm{~cm}^{-1}$, were severally originated from the stretching vibration and wagging vibration of $\mathrm{C}-\mathrm{H}$ bonds in methyl and methylene groups, the symmetrical deformation vibration of the $\mathrm{C}-\mathrm{H}$ bond in $\mathrm{Si}-\mathrm{CH}_{3}$, and the stretching vibration of the $\mathrm{Si}-\mathrm{C}$ bond. However, there were new strong double-shoulder peaks at $1134-1027 \mathrm{~cm}^{-1}$ in the PDMSQ spectrum rather than in the MTES and DTMS spectra. Those phenomena demonstrate that the hydrolysis-condensation reactions have been implemented and the inorganic skeleton of $\mathrm{Si}-\mathrm{O}-\mathrm{Si}$ has been formed. In addition, the tiny absorption peak at $3310 \mathrm{~cm}^{-1}$ was deduced from the signal of the incompletely-condensed Si-OH in the PDMSQ NPs curve. Thus, it is inferred that PDMSQ NPs have the expected chemical structure.

Figure 7 presents the ${ }^{29} \mathrm{Si}$ NMR spectrum of the PDMSQ NPs. It is obviously discovered that a pinnacle occurred at the chemical shift of $-67.7 \mathrm{ppm}$, which should be assigned to the characteristic peak of $\mathrm{Si}$ in the $\mathrm{T}^{3}$ structure of the PDMSQ as $\mathrm{RSi}(\mathrm{OSi})_{3}-[1,13,14]$. 


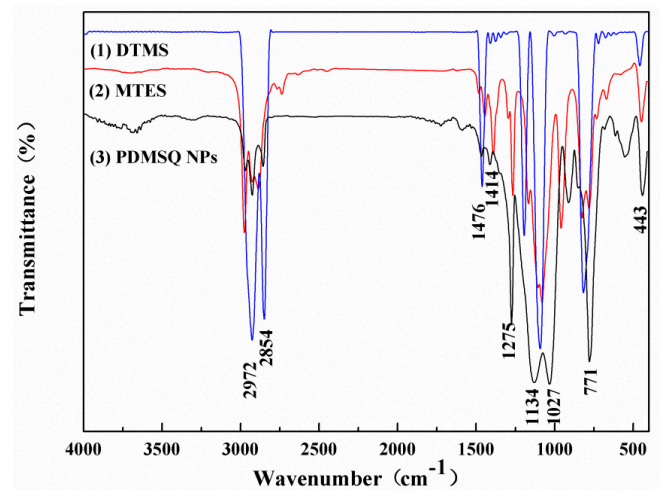

Figure 6. IR spectra of (1) DTMS, (2) MTES, and (3) the PDMSQ NPs sample P3.

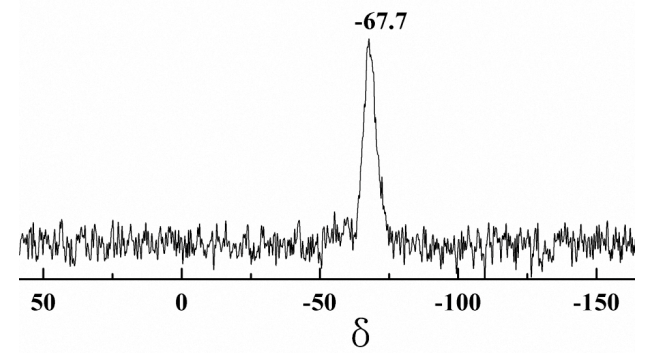

Figure 7. ${ }^{29} \mathrm{Si}$ NMR spectrum of the PDMSQ NPs sample P3.

In order to further confirm the surface chemical composition of the PDMSQ NPs, $\mathrm{X}$-ray photoelectron spectrometer (XPS) analysis was performed in this experiment and the results are shown in Figure 8, such as the XPS wide spectrum and Si2 $p$ high resolution spectrum. As shown in Figure 8a, there were three elements of carbon, oxygen and silicon on the PDMSQ NPs surface, atomic mass contents of which were $41.73 \mathrm{wt} . \%, 30.62 \mathrm{wt} . \%$ and $27.65 \mathrm{wt} . \%$, respectively, and the mass content of carbon was the largest. This indicates that the surface of PDMSQ NPs is covered by a large amount of methyl and methylene groups and it will be very helpful to reduce the surface free energy of the PDMSQ NPs surface. It is also detected from Figure $8 \mathrm{~b}$ of the Si2 $p$ high resolution spectrum that there were no characteristic absorption peaks of inorganic $\mathrm{SiO}_{2}$ and organic polysiloxane, except for the signal of Si2 $p$ in polysilsesquioxane at the binding energy of $103.34 \mathrm{eV}$. This result further confirms that the PDMSQ NPs have the expected chemical structure.

(a)

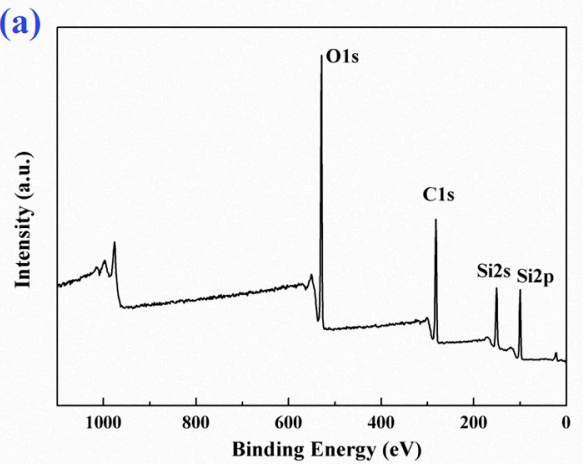

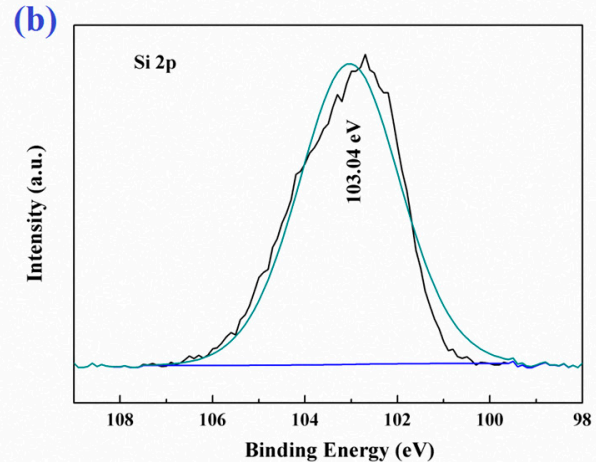

Figure 8. XPS survey spectrum (a) and high resolution Si2 $p$ spectrum (b) of sample P3.

\subsection{Thermal Analysis of the PDMSQ Nanoparticles}

Thermal stability is an important index to evaluate the application performance of polymer hybrid materials. Thus, thermogravimetric (TG) analysis was carried out to investigate the thermal stability of 
the PDMSQ nanosphere hybrid materials. The result is shown in Figure 9. It can be found that weight loss of the PDMSQ NPs was $5 \mathrm{wt} . \%$ when the temperature reached to $351{ }^{\circ} \mathrm{C}$, whereas, when the temperature continued to rise, the PDMSQ lost weight rapidly due to the cleavage of $\mathrm{C}-\mathrm{H}, \mathrm{C}-\mathrm{C}$ and $\mathrm{Si}-\mathrm{C}$ bonds [27]. Weight loss of the product attained half while the temperature was raised to $552{ }^{\circ} \mathrm{C}$. At this time, rate of thermal weight loss significantly decreased. When the temperature reached $678{ }^{\circ} \mathrm{C}$, the mass of the PDMSQ remained basically constant with a residue of $46.8 \%$, which was potentially considered as inorganic silica. Thus, on basis of the above statement, PDMSQ NPs should have excellent thermal stability.

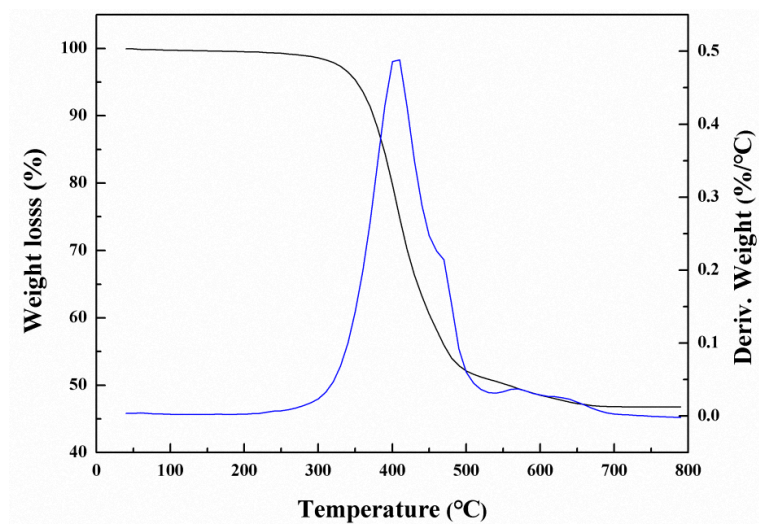

Figure 9. TGA of the PDMSQ NPs sample P3.

\section{6. $X R D$}

The XRD pattern of the PDMSQ NPs is shown in Figure 10 and, as seen in Figure 10, there was a narrower diffraction peak at $2 \theta=9.6^{\circ}$ and a broader diffraction peak at $2 \theta=21.4^{\circ}$, respectively. Those results demonstrate that the as-prepared PDMSQ NPs mainly present amorphous structures but also contain some small crystal structures due to spacing diffraction of the polysilsesquioxane chains [4].

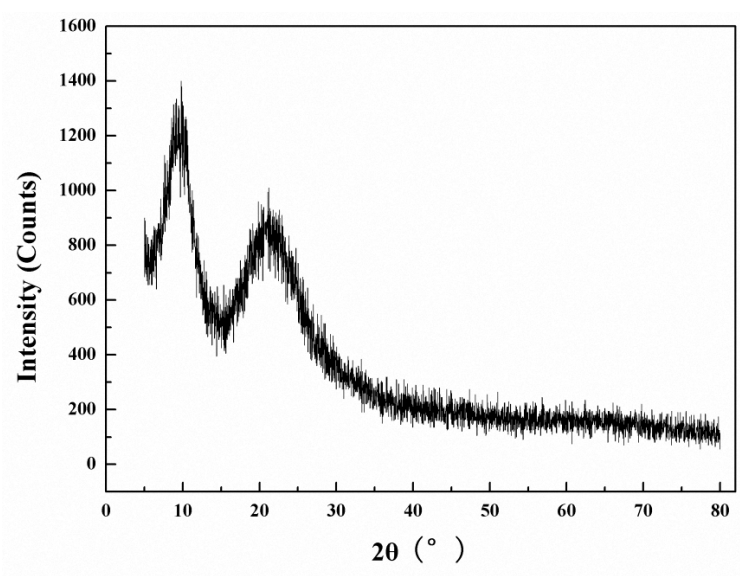

Figure 10. XRD of the PDMSQ NPs sample P3.

\subsection{Hydrophobicity of the PDMSQ Films}

Water contact angles (WCAs) on the PDMSQ films were measured and the results are presented in Table 2. It is clearly shown that the static WCAs on all the samples' films were larger than $150^{\circ}$ and the WCAs hysteresis were less than $10^{\circ}$. These consequences indicate that superhydrophobicity of those PDMSQ films has been achieved and thus those PDMSQ nanocomposite latexes show potential in the field of superhydrophobic coatings. 
Table 2. WCA results obtained for the PDMSQ films.

\begin{tabular}{ccccc}
\hline \multirow{2}{*}{ Samples Number } & \multicolumn{4}{c}{ WCA $\left(^{\circ}\right)$} \\
\cline { 2 - 5 } & Static & Advancing & Receding & Hysteresis \\
\hline P1 & 155.4 & 158.7 & 149.3 & 9.4 \\
P2 & 167.8 & 170.5 & 162.3 & 8.2 \\
P3 & 154.5 & 156.2 & 147.3 & 8.9 \\
P4 & 153.2 & 155.4 & 146.9 & 8.5 \\
P5 & 151.6 & 153.9 & 144.8 & 9.1 \\
P6 & 166.7 & 169.4 & 160.8 & 8.6 \\
P7 & 166.2 & 169.7 & 161.4 & 8.3 \\
P9 & 165.3 & 168.6 & 159.3 & 9.3 \\
P10 & 168.1 & 172.8 & 164.1 & 8.7 \\
\hline
\end{tabular}

\section{Conclusions}

A series of polydodecylmethylsilsesquioxane (PDMSQ) nanocomposite latexes have been prepared via emulsion polymerization of methyltriethoxysilane (MTES) and dodecyltrimethoxysilane (DTMS) and sodium hydroxide as the catalyst, and sodium dodecyl benzene sulfonate/Tween 80 as the mixed emulsifiers. PDMSQ NPs exhibited excellent thermal stability and were mainly amorphous but also contained some crystal structures. Importantly, the static WCAs on its latex films were larger than $150^{\circ}$ and the WCAs' hysteresis were less than $10^{\circ}$, thus those PDMSQ nanocomposite latexes show potential in the field of superhydrophobic coatings.

Author Contributions: Conceptualization, F.D. and W.X.; Methodology, F.D. and W.X.; Formal Analysis, F.D. and W.X.; Investigation, F.D. and W.X.; Data Curation, H.J.; Writing-Original Draft Preparation, F.D.; Writing-Review and Editing, W.X.; Supervision, W.X.; Project Administration, W.X. All authors have read and agreed to the published version of the manuscript.

Funding: This research was funded by the projects from the Key Program of Wenzhou (No. ZG2017028), the Basic Scientific Research Program of Wenzhou (No. G20190002), and the Doctorial Research Foundation of Shaanxi University of Science and Technology (No. BJ13-22), which provided financial support.

Conflicts of Interest: The authors declare no conflict of interest.

\section{References}

1. Dong, F.; Xie, H.; Zheng, Q.; Ha, C.-S. Superhydrophobic polysilsesquioxane/polystyrene microspheres with controllable morphology: From raspberry-like to flower-like structure. RSC Adv. 2017, 7, 6685-6690. [CrossRef]

2. Lee, Y.-G.; Park, J.-H.; Oh, C.; Oh, S.-G.; Kim, Y.C. Preparation of highly monodispersed hybrid silica spheres using a one-step sol-gel reaction in aqueous solution. Langmuir 2007, 23, 10875-10878. [CrossRef]

3. Yuan, J.; Ma, W.; Mo, J. Fabrication of highly monodisperse $\mathrm{CeO}_{2}$ @poly(methyl silsesquioxane) microspheres and their application in UV-shielding films. J. Appl. Polym. Sci. 2017, 134, 45065-45075. [CrossRef]

4. Kim, S.S.; Kim, V.; Kim, Y.B. Preparation and characterization of polysilsesquioxane particles containing UV-absorbing groups. Macromol. Res. 2012, 20, 437-446. [CrossRef]

5. Nagappan, S.; Ha, H.M.; Park, S.S.; Jo, N.-J.; Ha, C.-S. One-pot synthesis of multi-functional magnetite-polysilsesquioxane hybrid nanoparticles for the selective $\mathrm{Fe}^{3+}$ and some heavy metal ions adsorption. RSC Adv. 2017, 7, 19106-19116. [CrossRef]

6. Hao, L.F.; Yang, R.N.; Wang, X.C.; Xu, W.; An, Q.F. Progress on synthesis and application of polysissesquioxane micro/nanospheres. Fine Chem. 2017, 34, 1-10. (In Chinese)

7. Sankaraiah, S.; Lee, J.M.; Kim, J.H.; Choi, S.W. Preparation and characterization of surface-functionalized polysilsesquioxane hard spheres in aqueous medium. Macromolecules 2008, 41, 6195-6204. [CrossRef]

8. Hao, L.F.; Gao, T.T.; Wang, X.C.; Yang, B.Q.; Xu, W. Synthesis and characterization of polyaminopropyl methylsilsesquioxane nanospheres. Fine Chem. 2016, 33, 721-725. (In Chinese)

9. Ottenbrite, R.M.; Wall, J.S.; Sidiqui, J.A. Self-catalyzed synthesis of organo-silica nanoparticels. J. Am. Ceram. Soc. 2000, 83, 3214-3215. [CrossRef] 
10. Lee, D.; Kim, Y.-A.; Kim, J.-K.; Han, Y.-K.; Kim, Y.B. One step preparation of spherical silicon resins from immiscible reaction mixtures. Macromol. Res. 2008, 16, 353-359. [CrossRef]

11. Mori, H.; Sada, C.; Konno, T.; Koizumi, R.; Yonetake, K. Film-forming amphiphilic silsesquioxane hybrids prepared by hydrolytic co-condensation of hydroxyl-functionalized and fluorinated triethoxysilanes. Polymer 2012, 53, 3849-3860. [CrossRef]

12. Li, Z.; Tolbert, S.H.; Loy, D.A. Hybrid organic-inorganic menbrances from size exclusion deposition of fluorescent, octylene-bridged polysilsesquioxane partices. J. Non-Cryst. Solids 2014, 403, 88-96. [CrossRef]

13. Zou, F.; Yue, P.; Zheng, X.; Tang, D.; Fu, W.; Li, Z. Robust and superhydrophobic thiourethane bridged polysilsesquioxane aerogels as a potential thermal insulation material. J. Mater. Chem. A 2016, 4, 10801-10805. [CrossRef]

14. Ma, W.; Zhang, D.; Duan, Y.; Wang, H. Highly monodisperse polysilsesquioxane spheres: Synthesis and application in cotton fabrics. J. Colloid Interface Sci. 2013, 392, 194-200. [CrossRef]

15. Park, S.; Jung, S.; Heo, J.; Hong, J. Facile synthesis of polysilsesquioxane toward durable superhydrophilic/superhydrophobic coatings for medical devices. J. Ind. Eng. Chem. 2019, 77, 97-104. [CrossRef]

16. Schutzius, T.M.; Bayer, I.S.; Jursich, G.M.; Das, A.; Megaridis, C.M. Superhydrophobic-superhydrophilic binary micropatterns by localized thermal treatment of polyhedral oligomeric silsesquioxane (POSS)-silica films. Nanoscale 2012, 4, 5378-5385. [CrossRef]

17. Yuan, J.-J.; Kimitsuka, N.; Jin, R.-H. Bioinspired synthesis of a soft-nanofilament-based coating consisting of polysilsesquioxanes/polyamine and its divergent surface control. ACS Appl. Mater. Interfaces 2013, 5, 3126-3133. [CrossRef]

18. Xu, W.; Zhao, W.; Hao, L.; Wang, S.; Pei, M.; Wang, X. Synthesis and characterization of novel fluoroalkyl-terminated hyperbranched polyurethane latex. Appl. Surf. Sci. 2018, 436, 1104-1112. [CrossRef]

19. Xu, W.; Zhao, W.; Hao, L.; Wang, S.; Pei, M.; Wang, X. Synthesis of novel cationic fluoroalkyl-terminated hyperbranched polyurethane latex and morphology, physical properties of its latex film. Prog. Org. Coat. 2018, 121, 209-217. [CrossRef]

20. Osseo-Asare, K.; Arriagada, F. Growth kinetics of nanosize silica in a nonionic water-in-oil microemulsion: A reverse micellar pseudophase reaction model. J. Colloid Interface Sci. 1999, 218, 68-76. [CrossRef]

21. Mahbub, S.; Rub, M.A.; Hoque, A.; Khan, M.A.; Asiri, A.M. Critical micelle concentrations of sodium dodecyl sulfate and cetyltrimethylammonium bromide mixtures in binary mixtures of various salts at different temperatures and compositions. Russ. J. Phys. Chem. A 2019, 93, 2043-2052. [CrossRef]

22. Din, K.-U.; Siddiqui, U.S.; Kumar, S.; Dar, A.A. Micellization of monomeric and dimeric (gemini) surfactants in polar nonaqueous-water-mixed solvents. Colloid Polym. Sci. 2006, 284, 807-812. [CrossRef]

23. Das, C.; Das, B. Thermodynamic and interfacial adsorption studies on the micellar solutions of alkyltrimethylammonium bromides in ethylene glycol (1) + water (2) mixed solvent media. J. Chem. Eng. Data 2009, 54, 559-565. [CrossRef]

24. Ruiz, C.C.; Díaz-López, L.; Aguiar, J. Self-assembly of tetradecyltrimethylammonium bromide in glycerol aqueous mixtures: A thermodynamic and structural study. J. Colloid Interface Sci. 2007, 305, 293-300. [CrossRef]

25. Khoshnood, A.; Lukanov, B.; Firoozabadi, A. Temperature effect on micelle formation: Molecular thermodynamic model revisited. Langmuir 2016, 32, 2175-2183. [CrossRef] [PubMed]

26. Tsujii, K.; Saito, N.; Takeuchi, T. Krafft points of anionic surfactants and their mixtures with special attention to their applicability in hard water. J. Phys. Chem. 1980, 84, 2287-2291. [CrossRef]

27. Xu, W.; Hao, L.F.; An, Q.F.; Wang, X.C. Synthesis optimization of novel crosslinked polysiloxane with end-capped epoxy groups as water-proofing agent for fabric. Polym. Plast Technol. 2014, 53, 1682-1689. [CrossRef]

Publisher's Note: MDPI stays neutral with regard to jurisdictional claims in published maps and institutional affiliations. 